\title{
Uso de los medios digitales y su incidencia en la generación Y \& $\mathrm{Z}$
}

Use of digital media and its impact on generation $\mathrm{Y} \& \mathrm{Z}$

Marjorie Ordoñez Guartazaca

Fecha de recepción: 23 de abril de 2019

Fecha de aceptación: 10 de junio de 2019 


\title{
Uso de los medios digitales y su incidencia en la generación $Y \& Z$
}

Use of digital media and its impact on generation $\mathrm{Y} \& \mathrm{Z}$.

$$
\text { Marjorie Ordoñez Guartazaca }{ }^{1}
$$

Como citar: Ordoñez, M., (2019). Uso de los medios digitales y su incidencia en la generación Y \& Z, Revista Universidad de Guayaquil. 129(2), 2437. DOI: https://doi.org/10.53591/rug.v129i2.1370

\section{RESUMEN}

La presente investigación plantea cual es el comportamiento de la generación Y \& Z en la utilización de los medios digitales, y como esto influye en las nuevas generaciones a través de una cercana interacción con los estudiantes de la Universidad de Guayaquil de la Facultad de Ciencias Administrativas, con el objetivo de analizar su comportamiento al momento de utilizar los medios digitales. Se utilizó la metodología cuantitativa para el levantamiento de información a través de las encuestas. Los principales resultados demuestran la incidencia del uso de los medios digitales en ambas generaciones y su importancia en el desarrollo de la sociedad digital interconectada.

Palabras clave: Generación Y, Generación Z, Medios Digitales

\begin{abstract}
The present research raises what is the behavior of certain $\mathrm{Y} \& \mathrm{Z}$ generations in the use of digital media, and how this influences the new generations through close interaction with the students of the University of Guayaquil of the Faculty of Administrative Sciences, in order to analyze their behavior when using digital media. The quantitative methodology was used to gather information through the surveys. The main results show the incidence of the use of digital media in both generations and its importance in the development of the interconnected digital society.
\end{abstract}

Keywords: Generation Y, Generation Z, Digital Media

\footnotetext{
$1{ }^{3}$ Magister en Administración de Empresas. Docente en Facultad de Ciencias Administrativas de la Universidad de Guayaquil, Ecuad or, Correo electrónico: marjorie.ordonezg@ug.edu.ec
} 


\section{INTRODUCCIÓN}

El mundo es muy cambiante, y la tecnología evoluciona cada día, lo que hace que las generaciones poco a poco trasciendan, los niños de hoy nacen con nuevas características, con más conocimientos e inquietudes, en comparación con los niños de las generaciones pasadas, es por ello que se han creado diferentes tipos donde se clasifican por edad. La presente investigación estudia el comportamiento de la generación Y, conocidos como los Millennials nacidos en 1980 a 2000, y la generación Z, conocidos como post-Millennials, nacidos en 1995 a 2010 en plena era digital.

El análisis que se realizará nos permitirá inferir cuál es el comportamiento de las generaciones antes mencionadas en la utilización de los medios digitales, y cuáles son los factores que influyen en dicho comportamiento ya sean estos, económicos, culturales, o por las tendencias que se presentan continuamente, es decir, el consumo que las y los jóvenes hacen de las redes digitales, y concretamente de Internet, está transformando, no sólo el ocio y las formas de interacción de este segmento de la población con su entorno, sino, además, por extensión, de la sociedad en su conjunto. Puede decirse que, por primera vez, son los individuos más jóvenes los que toman la iniciativa, actuando como conductores en lo que se refiere al empleo de los medios y las funciones que emprenderán de forma intercomunicada.

El comportamiento y hábitos de consumo de la generación Millennials es un tema que está siendo muy estudiado porque son los futuros consumidores y el mercado no consigue aún saber cómo toman decisiones, cómo se informan y actúan en consecuencia. El ser humano es un ser social por naturaleza, rodeado por otros de sus mismos intereses y afinidades, donde puede poner en marcha los mecanismos y los recursos necesarios para desarrollar sus aptitudes y sus conocimientos.

Se escogió tratar este tema investigativo ya que es poco estudiado, actualmente se ve mucha diferencia en pensamientos de una persona nacida en Generación Y \& Z, se desea saber que similitudes y diferencias pueden tener en pensamiento y su uso de medios digitales. Se afirma que el desarrollo de la tecnología es fundamental para la formación de las personas se debe tener aún presente ciertas aplicaciones clásicas que se han dado en el pasar del tiempo, entre otras.

\section{Características de consumo de las generaciones $\mathrm{X}$ y $\mathrm{Y}$}

La generación Y o llamados Millennials son los nacidos entre 1988 y 2000. El Internet nació con ellos, por tanto, fueron creciendo juntos y su relación cada vez se tornó más íntima. Hoy los jóvenes Millennials son los principales propulsores del uso de Internet, hacen compras, investigan, se informan, se divierten y hasta buscan parejas en línea (García, 2017, p.2). 
Además, consumen contenido de entretenimiento $84 \%$ y de tecnología $72 \%$ como ver videos online, escuchar música streaming y ver series de TV streaming.

Ellos se han caracterizado por ser una generación dominante y muy controversial en todos los ámbitos que hacen presencia, ya que son volátiles, críticos y exigentes. Son muy visuales. Les encanta viajar y comprar. Son fanáticos de las compras en medios digitales, por lo que las empresas suelen hacerles seguimiento por vías virtuales. Tiene como ventaja que son multiplataforma, esto quiere decir que usan programas, sistemas o equipos que pueden funcionar de muchas maneras. (Gago, 2017, p.3)

Por otro lado, la Generación Z son los nacidos entre 1994 y 2010, son consumidores activos de tecnología y se perfilan como los mayores consumistas, aprecian los hobbies y los consideran como un estilo de vida. Son fieles a una marca específica y prefieren la innovación, sobre todo. La tecnología es parte de su vida, les facilita la manera de comunicarse y hacer parte de grupos específicos. Son altamente creativos, lo que hace que se desconcentren y se aburran fácilmente (Cerezo, 2016, p.98).

Para Iñaki (2017) los principales hallazgos de la generación Z son:

- Preferencia por dispositivos móviles, registrando 11 horas por semana, fuera del trabajo y correo electrónico.

- Son amantes de las redes sociales como Instagram y Snapchat, aunque la gran mayoría sigue usando Facebook.

- Son consumidores de contenido digital, usando Internet $23 \mathrm{~h}$ por semana para ver videos, programas de televisión, películas y escuchar podcasts.

- Más del 50\% de los encuestados revela que lo sitios web y las apps juegan un papel más importante en el proceso de compra que las búsquedas online, los anuncios de televisión o la publicidad en Internet. Las redes sociales se perfilan como el segundo canal más influyente.

- Les gusta recibir anuncios personalizados que proporcionen valor, especialmente si se trata de descuentos.

\section{Tendencias tecnológicas}

Las tendencias tecnologías infieren en nosotros, es un factor que nosotros no podemos prescindir porque influyen en la sociedad y somos nosotros quienes sufrimos los cambios, o somos el motor esencial del nacimiento de las tendencias tecnológicas (Price, 2017). Dentro de estas tendencias encontramos la inteligencia artificial, marketing digital, plataformas digitales, y la innovación tecnológica.

La inteligencia artificial es la capacidad que poseen las máquinas y la tecnología para emular 
aptitudes humanas como el razonamiento y la toma de decisiones gracias a un sistema de aprendizaje automático. Se espera que el desarrollo de esta tecnología tenga un impacto económico de \$15,7 billones para el 2020 (PwC) (Roca Salvatella, 2018).

El marketing digital según la revista (Marketing Digital, 2010):

Es un sistema interactivo dentro del conjunto de acciones de Marketing de la empresa, que utiliza los sistemas de comunicación telemáticos para conseguir el objetivo principal que marca cualquier actividad de marketing: Conseguir una respuesta mesurable ante un producto y una transacción comercial. Debido a la aparición de esta nueva modalidad comercial tan reciente no existe todavía una definición especifica ampliamente extendida entre la comunidad. "El marketing digital es una nueva forma comercial que lleva a cabo la empresa, utilizando la telemática. (p.2)

Las plataformas digitales, son espacios en Internet que permiten la ejecución de diversas aplicaciones o programas en un mismo lugar para satisfacer distintas necesidades. Cada una cuenta con funciones diferentes que ayudan a los usuarios a resolver distintos tipos de problemas de manera automatizada, usando menos recursos (Rockcontent, 2019), mediante cuatro tipos de plataformas: educativas, de comercio electrónico, especializadas.

Las plataformas educativas, se enfocan en la educación a distancia e intentan simular las mismas experiencias de aprendizaje que encontramos en un salón de clase. Sirven para complementar o sustituir el proceso de educación tradicional. Como ejemplos de plataformas educativas podemos mencionar a Blackboard, e-College y Moodle.

Las plataformas sociales o conocidas también como redes sociales, son muy utilizadas actualmente por gran parte de nuestra sociedad. Son aquellas plataformas digitales donde se guardan diversas informaciones relacionadas con las interacciones sociales. Gracias a ellas los usuarios se conectan y mantienen relaciones con familiares, amigos o conocidos a través de Internet. Podemos citar Facebook, LinkedIn, Instagram y Twitter como ejemplos de plataformas sociales.

Las plataformas de comercio electrónico, que ayudan al consumidor comprar diversos productos y servicios sin salir de casa, cada vez más las plataformas de comercio electrónico crecen en todos los sectores. Incluso en países donde esta práctica no era tan común hace algunos años, cada día se está popularizando más. Cómo ejemplos de plataformas de comercio electrónico tenemos a WooCommerce, Magento y Shopify.

Las plataformas especializadas que son creadas para satisfacer las necesidades de un grupo de usuarios. Existen las más diversas plataformas virtuales para todos los segmentos que podamos imaginar. Siempre que haya una necesidad, podrá crearse una plataforma para satisfacerla, 
independientemente del sector. Las más utilizadas en este sector son las plataformas de automatización de las estrategias de marketing y a continuación veremos un poco más sobre ellas.

Por último, la innovación tecnológica se refiere a la creación de un nuevo producto o servicio siempre dirigido a los consumidores. En otras palabras, lo que queremos decir es que existe innovación cuando las empresas presentan en el mercado nuevos elementos o, en su defecto, mejoran los ya existentes. Aunque esta innovación suele ir dirigida a la comercialización del producto o servicio también puede relacionarse con los procesos de producción dentro de la empresa. En definitiva, la innovación tiene que ver con el cambio dentro de la industria. (OBS Business School, 2017). Este término hace referencia a la creación de un producto o servicio nuevo dirigido a los consumidores, es la presentación de nuevos elementos en el mercado por parte de las empresas. Dentro de estos productos de innovación tecnológica se encuentran: la tecnología multimedia, tecnología cibernética, dispositivos electrónicos, medios de comunicación virtuales, redes sociales, sitios web, e internet.

\section{METODOS Y MATERIALES}

Se realizó una investigación cuantitativa, y de alcance descriptivo que permitió conocer la población de estudio de 9022 estudiantes de la Facultad de Ciencias Administrativas, cuyas proporciones se resumen en la Tabla 1. Aquí se visualiza que el 50\% de la población se encuentra concentrado en las carreras de Contaduría Pública Autorizado (Semestral), Ingeniería Comercial (Semestral), e Ingeniería en Marketing y Negociación (Semestral). De esta población, se toma una muestra aleatoria al 5\% de error dando un total de 369 estudiantes cuya proporción se muestra en la Tabla 1.

Tabla 1

Proporción de estudiantes por carrera

\begin{tabular}{lrrr}
\hline \multicolumn{1}{c}{ ESTRATOS } & \multicolumn{1}{c}{ N } & Porcentaje & n \\
\hline Administración de Empresas / Ingeniería Comercial (Semestral) & 2231 & $24.7 \%$ & 91 \\
Comercio Exterior/ Ingeniería en Comercio Exterior (Semestral) & 1027 & $11.4 \%$ & 42 \\
Gestión de la Información Gerencial/ Ingeniería en Sistemas Administrativos & 565 & $6.3 \%$ & 23 \\
Computacionales (Semestral) & 2232 & $24.7 \%$ & 91 \\
Contabilidad y Auditoría / Contaduría Pública Autorizado (Semestral) & 690 & $7.6 \%$ & 28 \\
Finanzas / Ingeniería en Tributación y Finanzas (Semestral) & 812 & $9.0 \%$ & 33 \\
Negocios Internacionales / Ingeniería en Gestión Empresarial (Semestral) & 1190 & $13.2 \%$ & 49 \\
Mercadotecnia/ Ingeniería en Marketing y Negociación (Semestral) & 275 & $3.0 \%$ & 11 \\
Turismo & $\mathbf{9 0 2 2}$ & $\mathbf{1 0 0 \%}$ & $\mathbf{3 6 9}$ \\
\hline Total & & & \\
\hline
\end{tabular}

Se realizó la estratificación en las Carreras de la Facultad de Ciencias Administrativas. se 
determinó la cantidad de estudiantes a encuestar aplicando el muestreo estratificado.

\section{RESULTADOS Y DISCUSIÓN}

Las principales carreras que cuentan con más estudiantes en la Facultad de Ciencias Administrativas son en su orden las siguientes: Contaduría Pública autorizada (CPA) donde concentra con el 19.14\%. Ingeniería Comercial con 18.45\%. Ingeniería de Marketing y Negociación con $9.84 \%$. Ingeniería en Comercio Exterior representando el $8 \%$ y el resto de las carreras influyendo $44.56 \%$.

\section{Hipótesis específica dimensión 1}

\section{Formulación de hipótesis estadística}

Hi: Las tendencias tecnológicas influyen en la Generación Y \& Z

Ho: Las tendencias tecnológicas no influyen en la generación Y \& Z

Tabla 2

Tabla cruzada Tendencias Tecnológicas*Generación $Y \& Z$

\begin{tabular}{|c|c|c|c|c|c|c|c|}
\hline & & & & Generación & $Y \& Z$ & & \\
\hline & & & $\begin{array}{l}\text { Totalmente de } \\
\text { acuerdo }\end{array}$ & $\begin{array}{c}\mathrm{De} \\
\text { acuerdo }\end{array}$ & Indeciso & $\begin{array}{c}\text { En } \\
\text { desacuerdo }\end{array}$ & Total \\
\hline & Totalmente de & Recuento & 15 & 48 & 6 & 1 & 70 \\
\hline & & $\begin{array}{l}\text { Recuento } \\
\text { esperado }\end{array}$ & 8,0 & 46,5 & 14,6 & 0,9 & 70,0 \\
\hline & & $\%$ del total & $4,1 \%$ & $13,0 \%$ & $1,6 \%$ & $0,3 \%$ & $19,0 \%$ \\
\hline & De acuerdo & Recuento & 24 & 171 & 45 & 0 & 240 \\
\hline & & $\begin{array}{l}\text { Recuento } \\
\text { esperado }\end{array}$ & 27,3 & 159,3 & 50,1 & 3,3 & 240,0 \\
\hline Tendencias & & $\%$ del total & $6,5 \%$ & $46,3 \%$ & $12,2 \%$ & $0,0 \%$ & $65,0 \%$ \\
\hline tecnológicas & Indeciso & Recuento & 3 & 23 & 25 & 2 & 53 \\
\hline & & $\begin{array}{l}\text { Recuento } \\
\text { esperado }\end{array}$ & 6,0 & 35,2 & 11,1 & 0,7 & 53,0 \\
\hline & & $\%$ del total & $0,8 \%$ & $6,2 \%$ & $6,8 \%$ & $0,5 \%$ & $14,4 \%$ \\
\hline & En desacuerdo & Recuento & 0 & 3 & 1 & 2 & 6 \\
\hline & & $\begin{array}{l}\text { Recuento } \\
\text { esperado }\end{array}$ & 0,7 & 4,0 & 1,3 & 0,1 & 6,0 \\
\hline & & $\%$ del total & $0,0 \%$ & $0,8 \%$ & $0,3 \%$ & $0,5 \%$ & $1,6 \%$ \\
\hline & & Recuento & 42 & 245 & 77 & 5 & 369 \\
\hline Total & & $\begin{array}{l}\text { Recuento } \\
\text { esperado }\end{array}$ & 42,0 & 245,0 & 77,0 & 5,0 & 369,0 \\
\hline & & $\%$ del total & $11,4 \%$ & $66,4 \%$ & $20,9 \%$ & $1,4 \%$ & $100,0 \%$ \\
\hline
\end{tabular}

Nota: Datos obtenidos de la base de datos de SPSS. 
Tabla 3

Prueba Chi-cuadradado hipótesis especifica 1

\begin{tabular}{lrcc}
\hline & Valor & df & Significación asintótica (bilateral) \\
\hline Chi-cuadrado de Pearson & $88.218^{\mathrm{a}}$ & 9 & 0.000 \\
Razón de verosimilitud & 51.577 & 9 & 0.000 \\
Asociación lineal por lineal & 35.676 & 1 & 0.000 \\
N de casos válidos & 369 & & \\
\hline
\end{tabular}

Nota: a. 7 casillas (43.8\%) han esperado un recuento menor que 5. El recuento mínimo esperado es .08.

Como el valor de significancia (valor critico observado) $0.000<0.05$ se rechaza la hipótesis nula y se acepta la hipótesis alternativa. es decir. las tendencias tecnológicas influyen en la Generación Y \& Z.

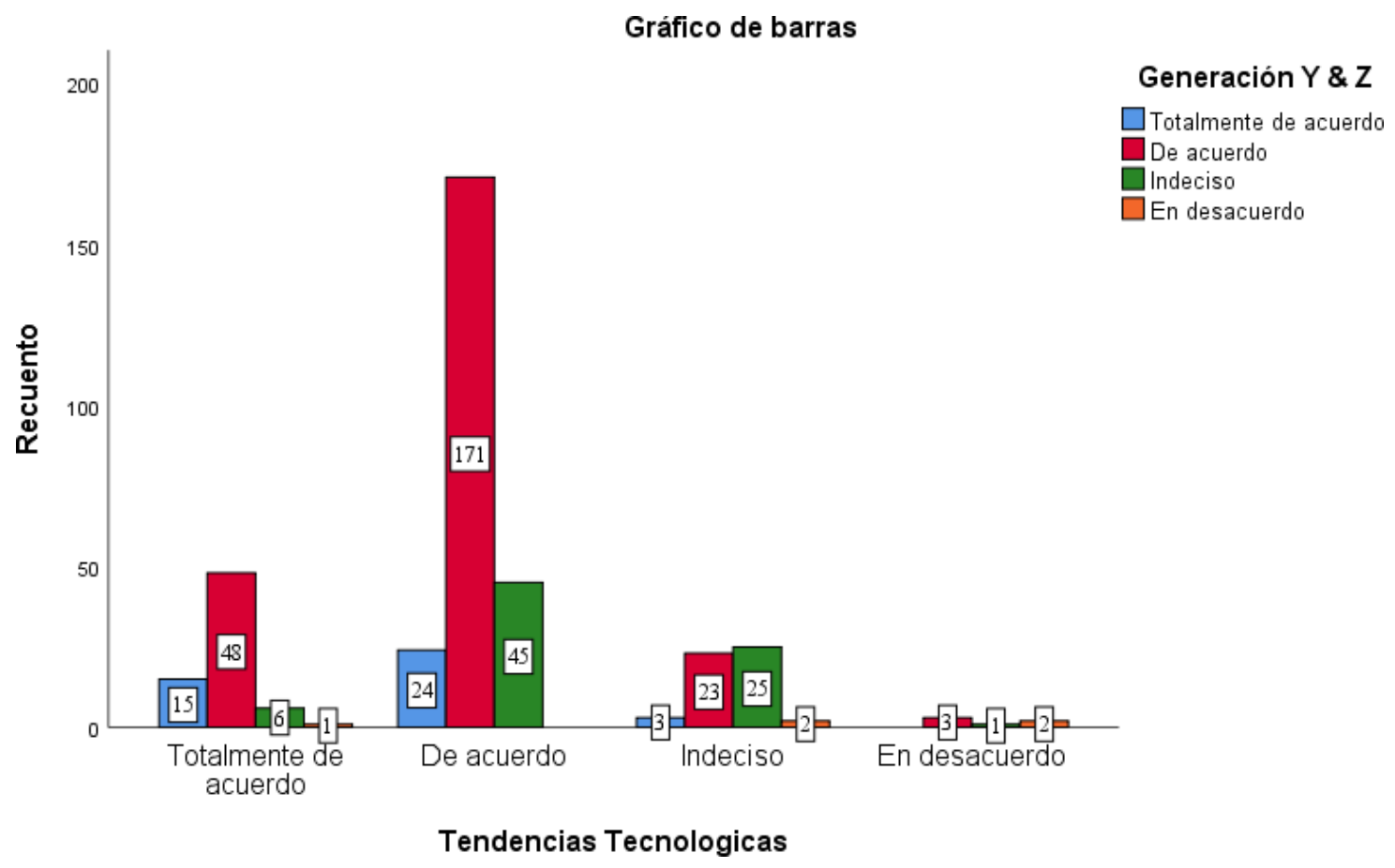

Figura 31. Contraste de la variable independiente con la dimensión 1

Existe una alta relación entre la variable independiente y con la hipótesis especifica “Tendencias tecnológicas" en la escala "De acuerdo" que representa un total de $46.3 \%$ de la población. es decir. 171 personas están de acuerdo que las tendencias tecnológicas influyen significativamente en la Generación Y \& Z.

\section{Hipótesis específica dimensión 2}

\section{Formulación de hipótesis estadística}

Hi: Las innovaciones tecnológicas inciden en la generación Y \& Z

Ho: Las innovaciones tecnológicas no inciden en la generación Y \& Z 
Tabla 4

Tabla cruzada Innovación Tecnológica*Generación $Y \& Z$

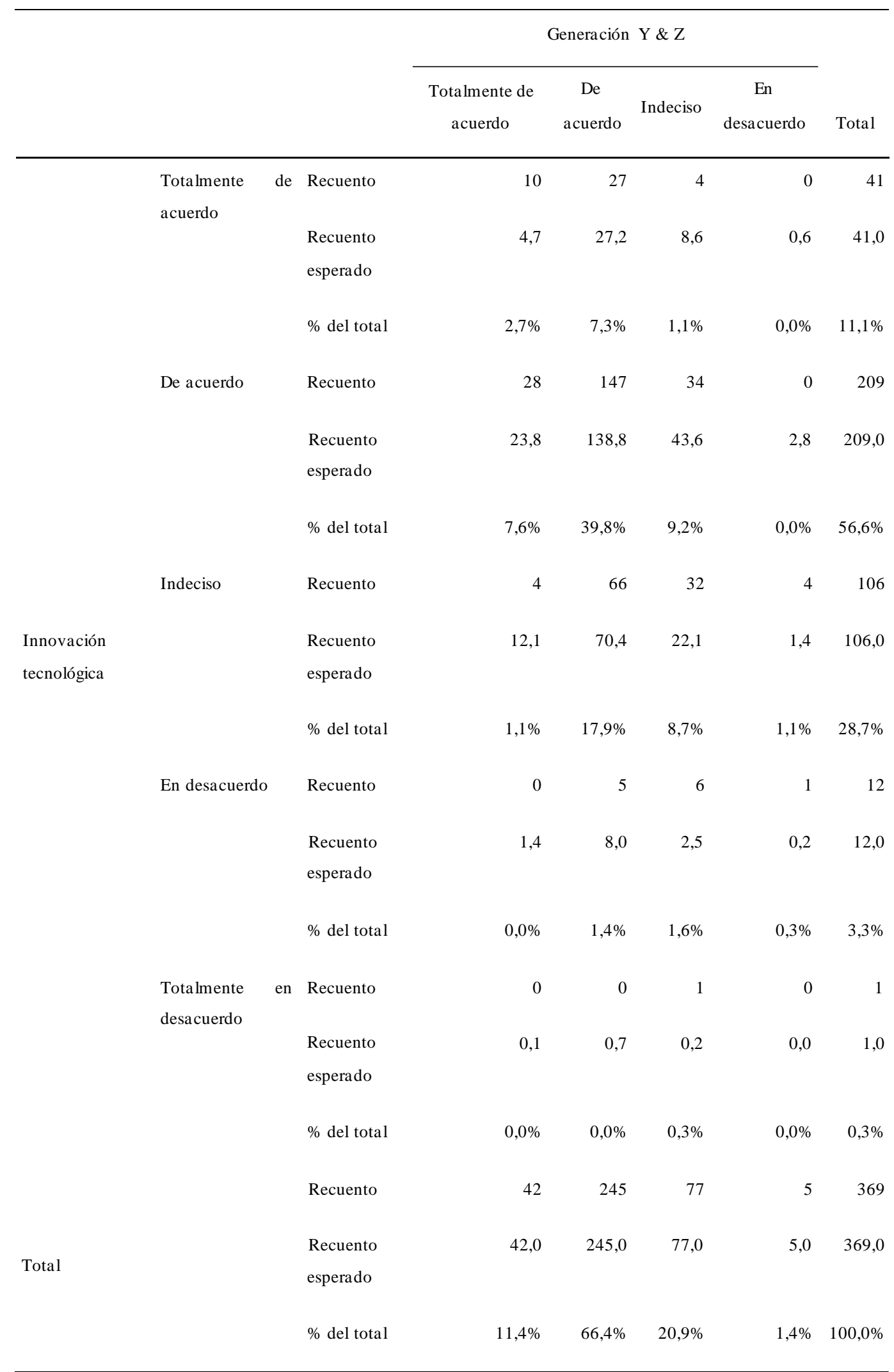

Nota: Datos obtenidos de la base de datos de SPSS. 
Tabla 5

Prueba Chi-cuadrado hipótesis específica 2

\begin{tabular}{lrrc}
\hline & Valor & Df & Significación asintótica (bilateral) \\
\hline Chi-cuadrado de Pearson & $45.373^{\mathrm{a}}$ & 12 & 0.000 \\
Razón de verosimilitud & 44.969 & 12 & 0.000 \\
Asociación lineal por lineal & 37.724 & 1 & 0.000 \\
N de casos válidos & 369 & \\
\hline Nota: a. 11 casillas (55.0\%) han esperado un recuento menor que 5. El recuento mínimo esperado es .01.
\end{tabular}

Como el valor de significancia (valor critico observado) $0.000<0.05$ se rechaza la hipótesis nula y se acepta la hipótesis alternativa. es decir. la innovación tecnológica incide en la Generación Y \& Z.

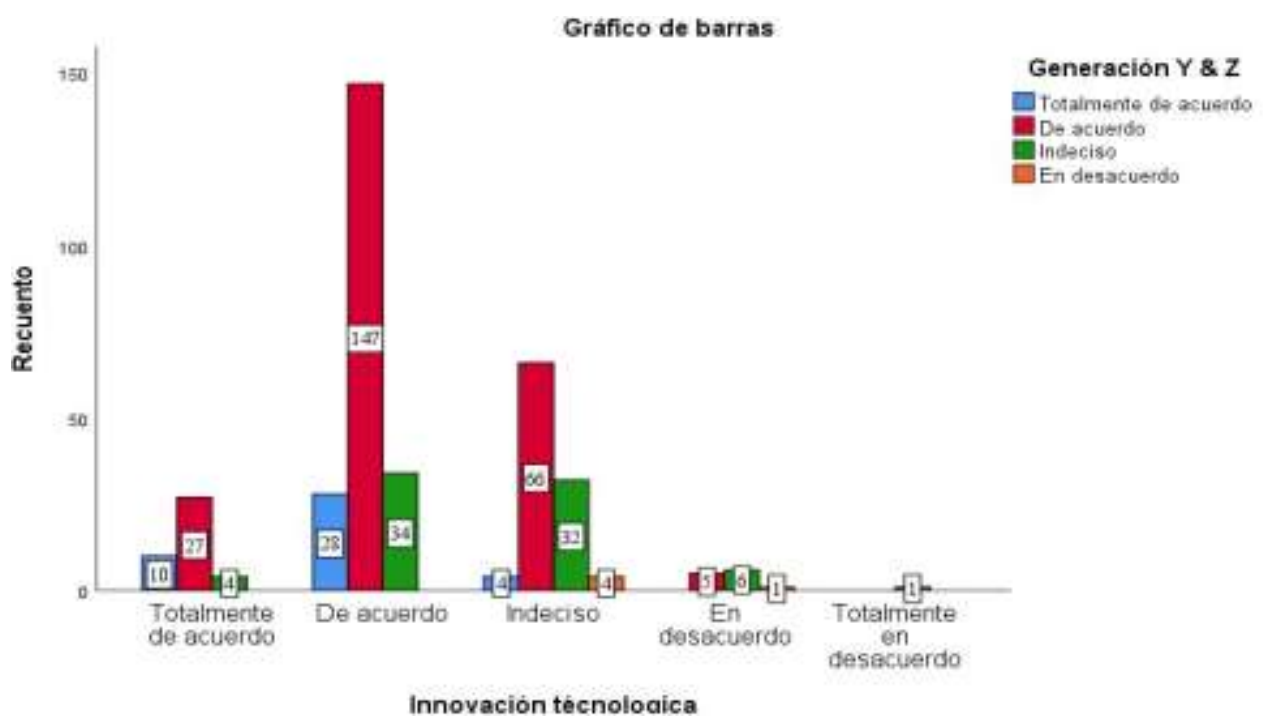

Figura 4. Contraste de la variable independiente con la dimensión 2

Existe una alta relación entre la variable independiente y con la hipótesis específica "Innovación tecnológica" en la escala "De acuerdo" que representa un total de $39.8 \%$ de la población. es decir. 147 personas están de acuerdo que la innovación tecnológica incide significativamente en la Generación Y \& Z.

\section{Hipótesis especifica dimensión 3}

\section{Formulación de hipótesis estadística}

Hi: Los medios de comunicación virtuales influyen en la Generación Y \& Z

Ho: Los medios de comunicación virtuales no influyen en la Generación Y \& Z 


\section{Tabla 6}

Tabla Crazada Medios de Comunicación*Generación $Y \& Z$

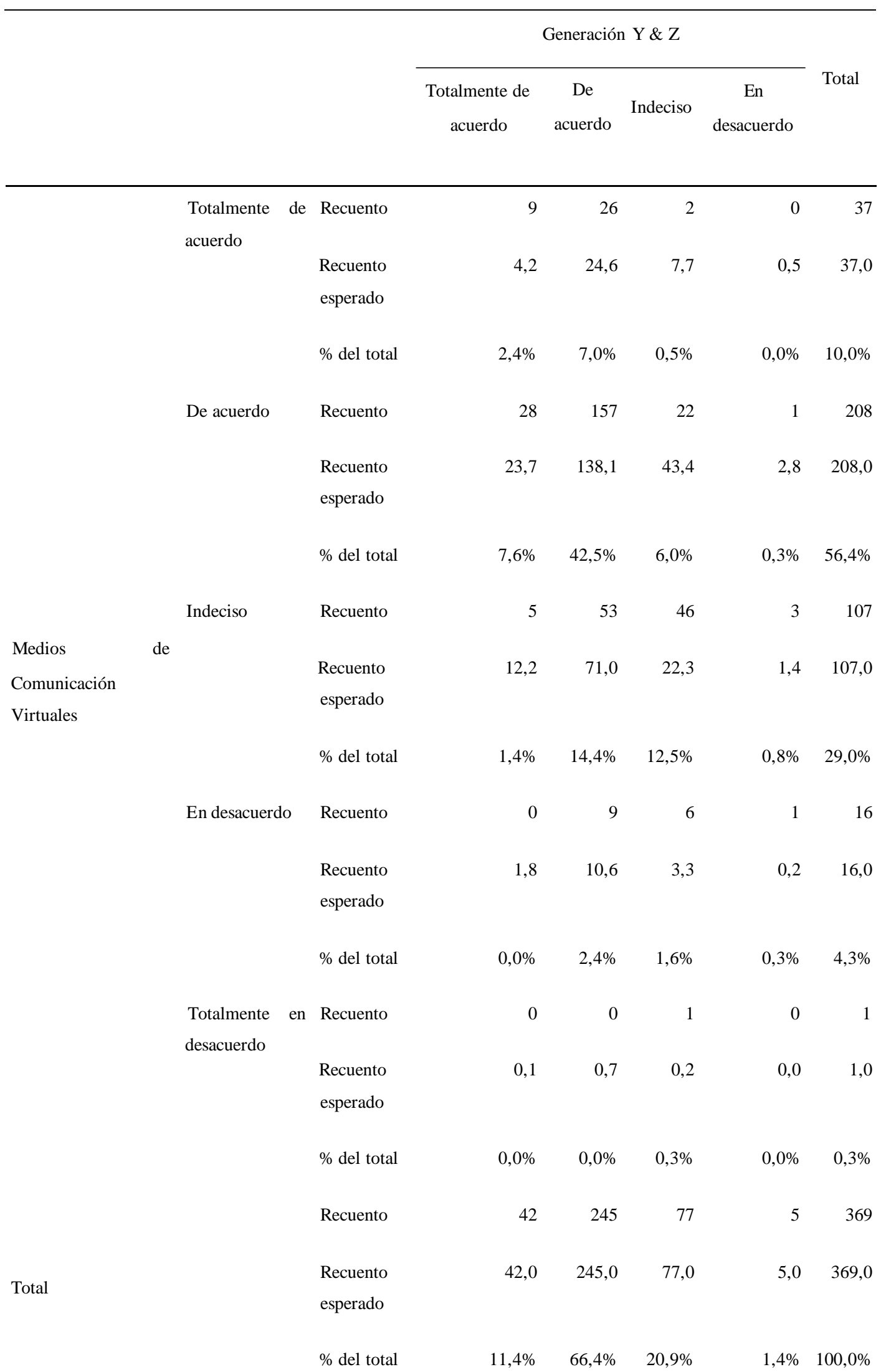


Tabla 7

Prueba Chi-cuadrado hipótesis específica 3

\begin{tabular}{lrrc}
\hline & Valor & Df & Significación asintótica(bilateral) \\
\hline Chi-cuadrado de Pearson & $71.754^{\mathrm{a}}$ & 12 & 0.000 \\
Razón de verosimilitud & 70.052 & 12 & 0.000 \\
Asociación lineal por lineal & 53.373 & 1 & 0.000 \\
N de casos válidos & 369 & \\
\hline Nota: a. 11 casillas $(55.0 \%)$ han esperado un recuento menor que 5. El recuento mínimo esperado es .01.
\end{tabular}

Como el valor de significancia (valor critico observado) $0.000<0.05$ se rechaza la hipótesis nula y se acepta la hipótesis alternativa. es decir. los medios de comunicación virtuales influyen en la Generación Y \& Z.

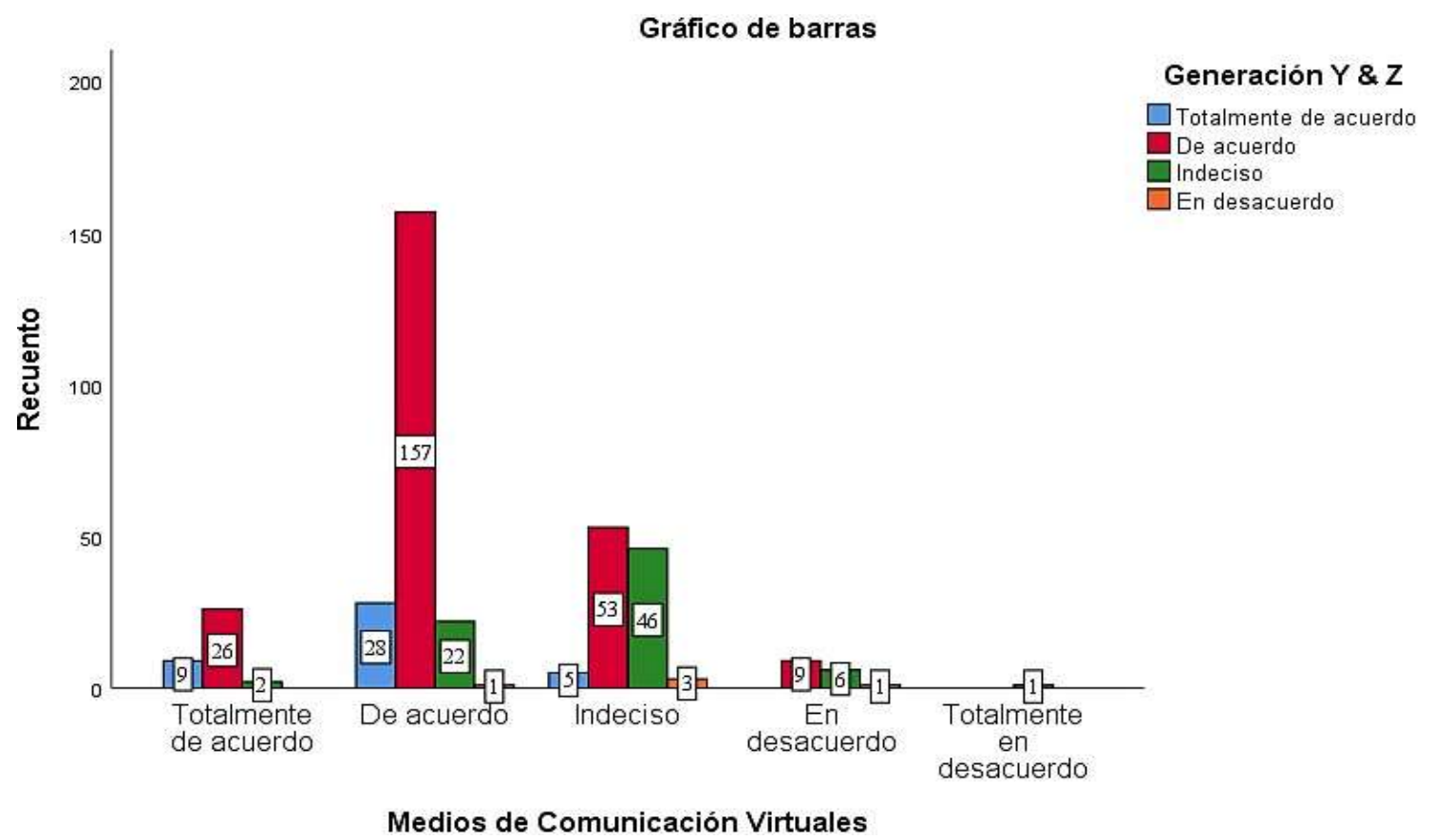

Figura. Contraste de la variable independiente con la dimensión 3

Existe una alta relación entre la variable independiente y con la hipótesis específica "Medios de Comunicación Virtuales" en la escala "De acuerdo" que representa un total de $42.5 \%$ de la población. es decir. 157 personas están de acuerdo que los medios de comunicación virtuales influyen significativamente en la Generación Y \& Z.

\section{Discusión}

En el artículo "Consumo de Medios de Comunicación digitales en Millennials ecuatorianos" escrito por Andrea V. Velásquez. Fanny Y. Paladines y Carlos V. Granda guarda similitud con el trabajo de investigación establecido sobre el "Uso de los medios digitales y su influencia en la Generación Y \& Z”.

En el artículo comprende el estudio del consumo y uso de los medios de comunicación - 
incluidos los medios digitales- en los adolescentes del Ecuador. lo cual obtuvo como resultado que el consumo cultural de los jóvenes se ha visto movilizado notablemente por el acceso a la red de redes. Internet. El estudio explica como en Ecuador 6 de cada 10 consumidores está en una red social. siendo Facebook que rodea el 62\%. Los jóvenes ecuatorianos. objeto de este estudio se adaptan al uso creciente de la comunicación digital. Cada vez más usan teléfonos inteligentes con conexión a Internet. El consumo de medios masivos a través de sus plataformas web o Apps. se ve incrementado considerablemente en 2015. (Velásquez, Paladines, \& Granda, 2017, pág. 375)

En comparación con el trabajo realizado se obtuvo que los estudiantes de la Facultad de Ciencias Administrativas de la Universidad de Guayaquil en la encuesta realizada. el dispositivo más utilizado por ambas generaciones es el Smartphone con un porcentaje del 78.32\%. en cuanto el 55.18\% de los participantes utilizan con mayor frecuencia WhatsApp esto se debe a que es una aplicación muy avanzada y de fácil manejo.

El objetivo de esta investigación es recopilar información sobre el comportamiento de la Generación Y \& Z. Las redes sociales son el medio a través del cual las existe relaciones con la Generación Z. A la hora de recopilar información con respecto a estas generaciones. el resultado ha sido fragmentado. en la que el estudio usa diferentes métodos para una buena comunicación con estas generaciones.

Dado el comportamiento de la generación Y no aceptan el lenguaje retorico e institucional. Es necesario conectar con una conversación bidireccional para involucrar a la generación Y en el proceso comunicativo honesto. La prescripción de terceros y el boca a boca influyen a esta generación más que en las predecesoras.

Por lo contrario. una importante mayoría señala tener un conocimiento avanzado en el uso de las redes sociales. donde el 55.18\% de los participantes utilizan con mayor frecuencia WhatsApp esto se debe a que es una aplicación muy avanzada y de fácil manejo.

Tal vez la única diferencia entre generaciones sea el criterio a nivel salarial. Ya que en la Generación $\mathrm{Z}$ no cuenta con niveles de ingresos muy altos ya que las fuentes de empleos hoy en día son muy escasas. El contexto socio- económico ecuatoriano puede ser aquí la variable que opere como explicativa: la constante sucesión de crisis económicas y frecuente afectación en condiciones laborales que afectan a los miembros de la generación Z.

\section{CONCLUSIONES}

La presente investigación se ha dedicado al estudio del comportamiento de la Generación Y \& $\mathrm{Z}$ y su incidencia en el uso de los medios digitales. se puede concluir según la información obtenida que. en la actualidad existe un choque generacional que se va dando de forma más 
notoria. a continuación. se presentan algunos de los resultados de forma detallada:

- La red social utilizada con mayor frecuencia es WhatsApp. obteniendo el $55.18 \%$ esto se debe a que es una aplicación muy avanzada y de fácil manejo. que no solo facilita la comunicación. sino que también permite el intercambio de documentos y archivos donde también el dispositivo más utilizado son los Smartphone.

- Se determinó que definitivamente la generación $\mathrm{Z}$ pertenece a la era digital. ya que en los resultados de datos se verificó que son las personas que gastan mayormente su tiempo en el uso de las redes sociales. y al momento de divertirse considera más importante el reunirse con sus amigos que con sus familiares.

- Finalmente se puede decir que la generación Y le cuesta adaptarse a las costumbres de las nuevas generaciones. mientras que $\operatorname{los} \mathrm{Z}$ se adaptan fácilmente debido a que tienen una percepción diferente sobre la vida.

Luego de realizar la investigación. se puede decir que la generación Z de los años (1994-2010) considerados nativos digitales utilizan más la tecnología que las generaciones anteriores (X y Y). por este medio se relacionan con el entorno a través de la web; en la actualidad todo se maneja a través de dispositivos tecnológicos y redes sociales. La última generación se caracteriza por tener amplia capacidad de adaptación en el uso de la tecnología. debido a que nacen en una era digital más avanzada donde la principal fuente de información es el internet a través de los medios digitales.

\section{REFERENCIAS}

Arroyo Galán. L. (2007). Multimedia móvil: empresa y sociedad. España: EOI Esc.Organiz.Industrial..

ASGECO. (2018). Dispositivos electrónicos. Obtenido de Asociación General de Consumidores: http://asgeco.org/consumeoriginal/dispositivos-electronicos/

Cardoso. G. (2010). Los medios de comunicacion en la sociedad en red / The Mass Media in the Networked Society: Filtros. escaparates y noticias / Filters. Windows and News. España: Editorial UOC.

Cerezo. P. (Diciembre de 2016). La Generación Z y la información. Estudios de Juventud.

97-108. Recuperado de

http://www.injuve.es/sites/default/files/2017/28/publicaciones/documentos_7._la_gen eracion_z_y_la_informacion.pdf

Chavéz. G. (09 de Mayo de 2017). GoDaddy. Obtenido de ¿Qué es un sitio web?: https://ar.godaddy.com/blog/que-es-un-sitio-web/

Durango. A. (2014). Las Redes Sociales. IT Campus Academy. 
Gago. S. (2017). "Millennials hijos de la era global". Tesis. Universidad de la Republica de Uruguay. Facultad de Psicologia . Recuperado de https://psico.edu.uy/presencias-enmedios/millennials-hijos-de-la-era-global

García Ayala. E. d. (29 de Junio de 2017). MILLENNIALS LA NUEVA GENERACIÓN DE PROFESIONISTAS DEL SIGLO XXI. Redalyc. 1-10.

Iñaki Ortega. N. V. (2017). Generación Z. Plataforma Editorial. 184. Recuperado de https://www.plataformaeditorial.com/libro/6086-generacion-z-

Madruga. A. (2008). La cibernética. Obtenido de Blogspot.com: http://lacibernetica.blogspot.com/2008/01/los-inicios-de-la-ciberntica.html Marketing Digital. (2010). Marketing digital. España: Editorial Vértice.

OBS Business School. (2017). OBS Business School;. Obtenido de Tendencias \& Innovación: https://www.obs-edu.com/int/blog-investigacion/sistemas/innovacion- tecnologica-tipos-ycaracteristicas-principales

Price. A. (2017). Tendecnias tecnológicas. Editorial Autores de Argentina. 7-15.

Roca Salvatella. (06 de Junio de 2018). RocaSalvatella. Obtenido de La "revolución" de las tendencias tecnológicas: https://rocasalvatella.com/blog-rs/la-revolucion-de-las- tendenciastecnologicas/

Rockcontent. (14 de Febrero de 2019). Rockcontent.com. Obtenido de Plataformas digitales: ¿qué son y qué tipos existen?: https://rockcontent.com/es/blog/plataformas-digitales/ Tendencias Digitales (27 de junio de 2017). Las generaciones y su uso de Internet.

Recuperado de https://tendenciasdigitales.com/las-generaciones-y-su-uso-de-internet/ Velásquez. A.. Paladines. F.. \& Granda. C. (2017). Consumo de Medios de Comunicación digitales en Millennials ecuatorianos. Memorias de la Décima Sexta Conferencia Iberoamericana en Sistemas. Cibernética e Informática (CISCI 2017). 373-378. 